\title{
Vitrification of preimplantation stages of mouse embryos
}

\author{
J. Ali ${ }^{*}$ and J. N. Shelton \\ Developmental Physiology Group, Division of Clinical Sciences, John Curtin School of Medical \\ Research, The Australian National University, PO Box 334, Canberra City, ACT 2601, Australia
}

\begin{abstract}
Three vitrification solutions (VS) namely VS1 $\left(5.5 \mathrm{~mol}\right.$ ethylene glycol $1^{-1}$ and $2.5 \mathrm{~mol}$ glycerol $\left.\mathrm{l}^{-1}\right)$, VS11 (6.0 mol ethylene glycol $\mathrm{l}^{-1}$ and $1.8 \mathrm{~mol}$ glycerol $\left.\mathrm{l}^{-1}\right)$ and VS14 $(5.5 \mathrm{~mol}$ ethylene glycol $\mathrm{l}^{-1}$ and $1 \mathrm{~mol}$ sucrose $\mathrm{l}^{-1}$ ) were tested for cryopreservation by vitrification of all developmental stages of mouse preimplantation embryos. In these experiments all preparative work was at room temperature $\left(25^{\circ} \mathrm{C}\right)$. VSI was toxic to embryos at and earlier than the eight-cell stage, whereas VSI1 was toxic to the four-cell and earlier stages. VS14 was the least toxic VS. All three VS resulted in good viability of vitrified Swiss Outbred day-4 embryos (morulae, early blastocysts and blastocysts) in vitro and vitrification with VS14 resulted in no loss of viability in all preimplantation stage Swiss Outbred embryos except one-cell embryos. One-cell $F_{1}$ embryos were vitrified successfully with VS14 and VS1. The minimal equilibration time essential for successful vitrification of embryos suggests that concentration of the intracellular solutes by dehydration has a major role in establishing conditions conducive to intracellular vitrification. Studies in vitro suggested that sucrose dilution was not necessary in the removal of cryoprotectant from vitrified eight-cell and day-4 mouse embryos but, in contrast, development of vitrified day-4 embryos in vivo was better when the VS was diluted with sucrose. When VSI or VSII was removed from vitrified embryos with sucrose the number of live fetuses obtained after transfer to surrogates did not differ from the number obtained from untreated embryos. Vitrification was not teratogenic; all mice that developed from vitrified embryos appeared normal and later reproduced normally. The present study demonstrated that vitrification can be routinely used to cryopreserve mouse embryos with no loss of viability.
\end{abstract}

\section{Introduction}

The relevance of vitrification to cryopreservation of biological material was first suggested by Luyet (1937), but it was not fully appreciated as a practical strategy for the cryopreservation of embryos until recently. Vitrification circumvents intracellular ice formation, which is a major cause of cell death, but cryoprotectant toxicity and osmotic injury to embryos can occur. These effects depend on the choice of cryoprotectant, concentration of cryoprotectant, duration of exposure and temperature of exposure. Rall and Fahy (1985), using a mixture of four cryoprotectants and stepwise exposure of the embryos at a temperature of $4{ }^{\circ} \mathrm{C}$, showed that mouse embryos could be successfully cryopreserved by vitrification. Scheffen et al. (1986), after tests on combinations of the two cryoprotectants for toxicity and capacity to vitrify, reported a simpler procedure using glycerol and propylene glycol at room temperature. However, this also involved the two-step exposure of embryos to the vitrifying medium. Kasai et al. (1990) obtained excellent results after onestep exposure of embryos to a vitrification medium containing only ethylene glycol as a permeating cryoprotectant plus Ficoll and sucrose.

*Present address: IVF Laboratory, Department of Obstetrics and Gynaecology, National University Hospital, Singapore 0511.

Received 18 August 1992.
The aim of the research described here was to develop a method for cryopreservation by vitrification that can be performed at room temperature for all developmental stages of the preimplantation mouse embryo with minimal or no loss of viability. In preliminary studies over 6000 combinations of cryoprotectants were investigated to identify mixtures that would vitrify on plunging into liquid nitrogen and remain ice-free on warming (Ali, 1992). Subsequent studies on embryotoxicity led to the formulation of three promising vitrification solutions (VS), namely, VS1 $\left(5.5 \mathrm{~mol}\right.$ ethylene glycol $\mathrm{l}^{-1}$ and $2.5 \mathrm{~mol}$ glycerol $\left.1^{-1}\right)$, VSII $\left(6.0 \mathrm{~mol}\right.$ ethylene glycol $1^{-1}$ and $\left.1.8 \mathrm{~mol}_{\text {glycerol }} \mathrm{1}^{-1}\right)$ and VS14 (5.5 mol ethylene glycol $\mathrm{l}^{-1}$ and $1.0 \mathrm{~mol}$ sucrose $\left.1^{-1}\right)$. The efficacy of the three vitrification solutions was tested by the viability of vitrified embryos in culture in vitro and after transfer to pseudopregnant surrogates. Experiments were also performed to determine the need for sucrose dilution of vitrification solution from embryos after warming.

\section{Materials and Methods}

\section{Generation and collection of embryos}

Mouse embryos of all developmental stages were obtained without superovulation. Swiss Outbred (SO) embryos were 
used in most experiments. Embryos with $\mathrm{F}_{1}$ cytoplasm ((C57BL/ $6 \times \mathrm{SJL}) \times \mathrm{SO}$ ) were used for a limited number of experiments. For convenience these will be referred to as $F_{1}$ embryos. The oestrous cycles of donor females were synchronized by pheromonal stimulation (Whitten, 1957). The group of females was exposed to a male separated by a wire grid for $48 \mathrm{~h}$ to synchronize their oestrous cycles. After this period each female was placed with one male and the following morning the female mice were examined for copulation plugs. The mice were subjected to a regimen of $14 \mathrm{~h}$ light:10 h dark with the light period extending from 08:00 to 22:00 h. The day copulation was detected was considered as day I of pregnancy. One-cell embryos were collected at 18:00-20:00 $\mathrm{h}$ on day $\mathrm{I}$; two-cell embryos at 09:00-10:00 $\mathrm{h}$ on day 2; four-cell embryos at 05:00 to $06: 00 \mathrm{~h}$ on day 3; eight-cell embryos at 10:00-11:00 h on day 3; compacted morulae at 06:00-07:00 h on day 4; early blastocysts at 09:00-10:00 h on day 4 and blastocysts at 11:00$12: 00 \mathrm{~h}$ on day 4. Recovery of embryos was performed as described by Hammond (1949). Recovered embryos were pooled in small droplets of Hepes-buffered Whitten's medium (HWM) under paraffin oil. HWM consisted of Whitten's medium (Whitten, 1971) containing $25 \mathrm{mmol}$ sodium Hepes $\mathrm{I}^{-1}$ and $4.0 \mathrm{mmol}$ sodium bicarbonate $\mathrm{l}^{-1}$ with the $\mathrm{pH}$ adjusted to 7.25 at room temperature. Embryos of poor quality were discarded and the remaining embryos were apportioned equally as appropriate for individual experiments.

\section{Vitrification of embryos}

Mouse embryos were either exposed at $25^{\circ} \mathrm{C}$ to one of the three VS for 1-3 min (one- to eight-cell embryos and blastocysts were exposed for $1 \mathrm{~min}$; early blastocysts for $2 \mathrm{~min}$; morulae for $3 \mathrm{~min}$ ) or vitrified with VS1, VS11 or VS14. These durations were selected after toxicity studies (Ali, 1992) showed that at $25^{\circ} \mathrm{C}$ one- to eight-cell embryos were affected by quite brief exposure to VS11. Two-cell embryos showed toxic effects after even $1 \mathrm{~min}$ of exposure and blastocysts showed slight toxic effects after $5 \mathrm{~min}$, whereas morulae were more tolerant. Embryos to be vitrified were exposed to the vitrification solution as described above and within this period the embryos were loaded into $0.25 \mathrm{ml}$ plastic insemination straws (I.M.V., L'Aigle). Each straw was prepared by filling with VS and the plug end was heat sealed. With the VS-filled straw held horizontally, embryos (average of 12 per straw) were introduced by a fine pipette $35-40 \mathrm{~mm}$ from the open end of the straw which was then heat sealed. The sealed straw was then plunged horizontally into liquid nitrogen and held below the surface for $10-20 \mathrm{~s}$ (cooling rate about $2500^{\circ} \mathrm{C} \mathrm{min}^{-1}$; Rall, 1987). The straws containing vitrified embryos were stored in liquid nitrogen until they were used.

\section{Warming and culture of embryos}

The embryos were warmed by directly immersing the vitrified straws into a water bath maintained at $25^{\circ} \mathrm{C}$ where they were held for 6-10 s to allow complete warming to $25^{\circ} \mathrm{C}$ (warming rate about $1000^{\circ} \mathrm{C} \mathrm{min}^{-1}$; Rall et al., 1986). The contents of the straw were emptied into $1.0 \mathrm{ml}$ of $1.0 \mathrm{~mol}$ sucrose $1^{-1}$ and stirred gently to facilitate mixing of the two solutions.
The embryos were quickly visualized under a microscope, picked up with a capillary pipette and transferred to a $100 \mu \mathrm{l}$ drop of $1.0 \mathrm{~mol}$ sucrose $\mathrm{l}^{-1}$ under paraffin at $25^{\circ} \mathrm{C}$. The cryoprotectants were diluted in $1.0 \mathrm{~mol}$ sucrose $\mathrm{l}^{-1}$ for $10 \mathrm{~min}$ and finally in HWM for $5 \mathrm{~min}$ before the embryos were used in various experiments. The cryoprotectants in the embryos that were exposed to VS but not vitrified were removed as described above. The embryos were finally transferred to Whitten's medium (WM) and cultured up to the blastocyst stage at $37^{\circ} \mathrm{C}$ in an atmosphere of $5 \% \mathrm{CO}_{2}$ in air.

\section{Experiments}

Experiment 1: vitrification of preimplantation stages of mouse embryos. All preimplantation stages of $\mathrm{SO}$ and one-cell $\mathrm{F}_{1}$ embryos were exposed to or vitrified with all three VS. After warming and dilution with $1 \mathrm{~mol}$ sucrose $1^{-1}$, the vitrified embryos were cultured in WM up to the blastocyst or hatching blastocyst stages.

Experiment 2: effect of dilution of VS with and without sucrose. All preimplantation stages of the $\mathrm{SO}$ embryos were vitrified with VS11. After warming, the VS was diluted with or without sucrose (direct dilution with medium). For dilution of VS without sucrose, the embryos were placed in $0.1 \mathrm{ml}$ droplets of HWM under paraffin oil for $5 \mathrm{~min}$ at $25^{\circ} \mathrm{C}$. The embryos were then washed twice in HWM before culture in WM. The in vitro survival of treated embryos diluted with or without sucrose was compared with survival of untreated embryos.

Experiment 3: viability of day-4 $S O$ and $F_{1}$ embryos when transferred to surrogates. Fresh and vitrified day- $4 \mathrm{SO}$ and $\mathrm{F}_{1}$ mouse embryos were transferred to surrogates at day 3 of pseudopregnancy as described by McLaren and Michie (1956) with minor modifications. The mice were anaesthetized with an inhalation anaesthetic, methoxyfluorane (Penthrane: Abbott Laboratories, North Chicago). The surrogates were anaesthetized by placing them in a desiccator (11 capacity) containing a ball of cotton wool saturated with $1.5 \mathrm{ml}$ anaesthetic. The anaesthetized mouse was surgically prepared for transfer by the first operator while a second operator picked up the embryos in a fine pipette attached via a plastic tube to a syringe controlled by a syringe driver. The loaded pipette was handed to the first operator who inserted it into the uterine lumen via a puncture hole. The embryos were expelled by the second operator turning the syringe driver. The first author's sensitivity to mice necessitated the wearing of a mask and precluded transfer by mouth operated pipette. The VS in SO embryos vitrified with VS1 and VS11 were diluted with and without sucrose. The VS in $F_{1}$ embryos vitrified with VS11 and in SO embryos vitrified with VS14 were diluted with sucrose. After warming and dilution of VS the vitrified embryos were cultured in WM for 45-60 min before transfer. Embryo survival in surrogates was determined by dissection on day 18 of pregnancy or after parturition.

Experiment 4: reproductive capability of mice derived from vitrified embryos. SO mice derived from vitrified (VS1 and VS11) day-4 embryos after transfer to surrogates were used for this experiment. Five randomly selected pairs ( 1 male and 1 female) 
Table 1. Percentage survival of preimplantation stages of Swiss Outbred, and one-cell stage of $F_{1}$ mouse embryos exposed to or vitrified in vitrification solutions (VS)

\begin{tabular}{|c|c|c|c|c|c|c|c|c|c|}
\hline \multirow[b]{2}{*}{ VS } & \multirow[b]{2}{*}{$\begin{array}{l}\text { Embryo } \\
\text { treatment }\end{array}$} & \multicolumn{8}{|c|}{ Developmental stage of embryos } \\
\hline & & Blastocyst & $\begin{array}{c}\text { Early } \\
\text { blastocyst }\end{array}$ & Morulae & 8-cell & 4-cell & 2-cell & I-cell & $F_{1}$ 1-cell \\
\hline \multirow[t]{3}{*}{ VSI } & Control & $99(88)^{* *}$ & $93(86)$ & $80(49)$ & $87(39)$ & $83(24)$ & $86(51)$ & $40(40)$ & $87(31)$ \\
\hline & Exposed & $95(87)$ & $95(84)$ & $80(51)$ & $37(38)^{\mathrm{d}}$ & $0(24)$ & $12(51)^{d}$ & $7(41)^{c}$ & $78(32)$ \\
\hline & Vitrified & $93(86)$ & $95(86)$ & $88(58)$ & $22(37)^{d}$ & $0(24)$ & $O(53)$ & $0(41)$ & $67(30)$ \\
\hline \multirow[t]{3}{*}{ VS11 } & Control & $100(68)$ & $100(58)$ & $100(42)$ & $95(56)$ & $96(27)$ & $86(51)$ & $40(40)$ & $91(22)$ \\
\hline & Exposed & $97(69)$ & $100(58)$ & $100(42)$ & $75(57)^{b}$ & $41(27)^{d}$ & $42(52)^{d}$ & $37(43)$ & $86(22)$ \\
\hline & Vitrified & $93(76)$ & $96(56)$ & $93(45)$ & $91(57)$ & $12(26)^{d}$ & $23(53)^{d}$ & $16(43)^{a}$ & $0(22)$ \\
\hline \multirow[t]{3}{*}{ VS14 } & Control & $100(35)$ & $100(39)$ & $98(48)$ & $90(69)$ & $81(36)$ & $86(51)$ & $46(37)$ & $91(23)$ \\
\hline & Exposed & $100(35)$ & $97(38)$ & $100(48)$ & $97(69)$ & $86(36)$ & $81(37)$ & $41(37)$ & $96(23)$ \\
\hline & Vitrified & $97(36)$ & $97(38)$ & $98(48)$ & $87(55)$ & $70(33)$ & $71(51)$ & $19(36)^{\mathrm{a}}$ & $92(24)$ \\
\hline
\end{tabular}

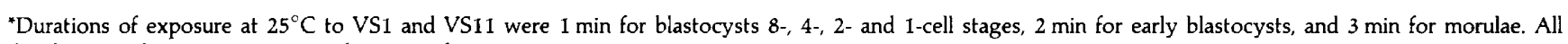
developmental stages were exposed to VS14 for $2 \mathrm{~min}$.

**Numbers in parentheses are numbers of embryos in group.

$\mathrm{F}_{1}$ embryos were $((\mathrm{C} 57 \mathrm{BL} / 6 \times \mathrm{SJL}) \times$ Swiss Outbred $)$.

a $P<0.05$ compared with controls.

${ }^{b} P<0.01$ compared with controls.

$s p<0.005$ compared with controls.

d $P<0.0001$ compared with controls.

were placed in individual cages and their subsequent litters were examined for abnormality.

\section{Statistical analysis}

Chi-square tests were used to compare results between groups when the number of samples exceeded 25. For fewer samples Fisher's exact test was used.

\section{Results}

\section{Experiment 1: vitrification of preimplantation stages of mouse embryos}

The survival of day-4 SO mouse embryos (blastocysts, early blastocysts and morulae) that were vitrified with VS1, VS11 and VS14 was comparable to that of control embryos (Table 1).

The viability of eight-cell embryos was reduced by exposure to and vitrification with VSI and by exposure to, but not vitrification with, VS11. Viability was not affected by VS14. Fourand two-cell embryos were of reduced viability after exposure to VS1 and VS11 but not to VS14. One-cell SO embryos were adversely affected by exposure to VSI, and by vitrification with VS1, VS11 and VS14. The viability of one-cell $F_{1}$ embryos was reduced only by vitrification with VS11.

\section{Experiment 2: effect of dilution of VS with and without sucrose}

The survival of vitrified (VS11) day-4 embryos (blastocysts, early blastocysts and morulae) and eight-cell embryos was not affected when the VS was diluted with sucrose or without sucrose (that is directly with HWM) (Table 2). Survival of fourcell and two-cell embryos was reduced significantly by vitrifi-
Table 2. Percentage survival of preimplantation stages of Swiss Outbred mouse embryos after vitrification* with VSII and dilution with and without sucrose

\begin{tabular}{lccc}
\hline $\begin{array}{l}\text { Stage of } \\
\text { development }\end{array}$ & $\begin{array}{c}\text { Untreated } \\
\text { controls }\end{array}$ & $\begin{array}{c}\text { Diluted } \\
\text { with medium }\end{array}$ & $\begin{array}{c}\text { Diluted with } \\
\text { 1 mol sucrose } \mathrm{I}^{-1}\end{array}$ \\
\hline Blastocysts & $100(15)^{* *}$ & $93(15)$ & $79(15)$ \\
$\begin{array}{l}\text { Early } \\
\quad \text { blastocysts }\end{array}$ & $100(27)$ & $100(24)$ & $100(23)$ \\
$\begin{array}{l}\text { Morulae } \\
\text { 8-cell }\end{array}$ & $86(14)$ & $92(13)$ & $85(13)$ \\
4-cell & $98(52)$ & $88(34)$ & $91(55)$ \\
2-cell & $95(19)$ & $28(15)^{b}$ & $17(18)^{\mathrm{c}}$ \\
I-cell & $87(15)$ & $44(16)^{\mathrm{a}}$ & $0(16)$ \\
& $43(14)$ & $0(15)$ & $0(15)$ \\
\hline
\end{tabular}

"Durations of exposure to VS11 before vitrification were 1 min for blastocysts, 8-, 4-, 2- and I-cell embryos, 2 min for early blastocysts and 3-min for morulae. ${ }^{* *}$ Numbers in parentheses are numbers of embryos in the group.

${ }^{a} P<0.05$ compared with controls.

${ }^{b} p<0.001$ compared with controls.

c $P<0.0001$ compared with controls.

cation; but the survival of two-cell embryos was significantly higher when diluted with medium than when diluted with sucrose. None of the vitrified one-cell embryos survived irrespective of the dilution procedure used.

Experiment 3: viability of day-4 SO and $F_{1}$ embryos when transferred to surrogates

The total number of implantations of day-4 SO embryos vitrified with VSI or VSII was significantly greater when the 
Table 3. The effect of VS and method of VS dilution on survival of vitrified day-4 Swiss Outbred mouse embryos when transferred to pseudopregnant C57BL/6 surrogates

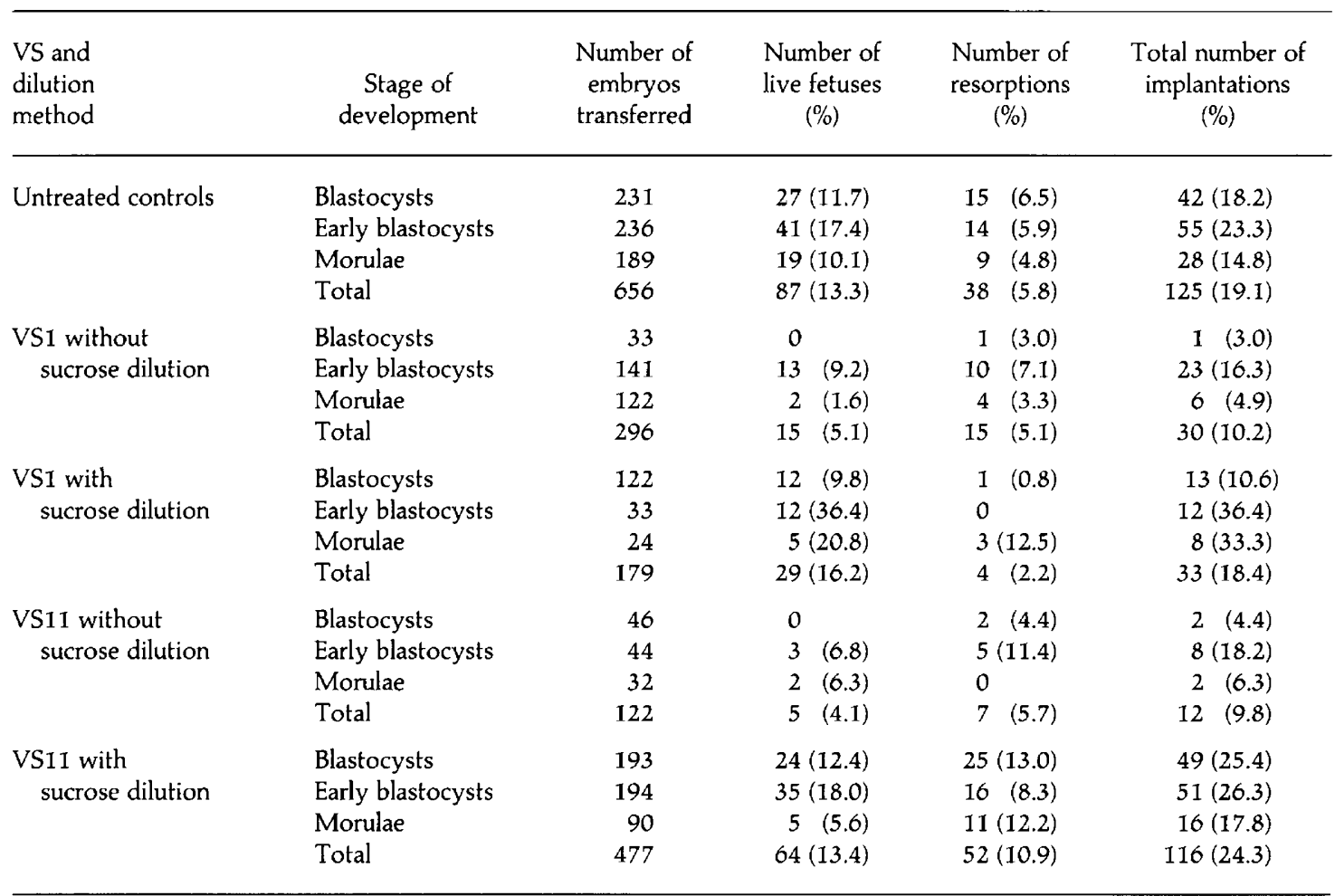

Surnmary of statistical analysis: (i) Total number of implantations: VSI with sucrose was significantly better than VSI without sucrose $(P<0.05)$; VS11 with sucrose was significantly better than VS11 without sucrose $(P<0.001)$; VS1I was significantly better than VSI $(P<0.001)$; VS11 was significantly better than controls $(P<0.05)$; VS11 with sucrose dilution was significantly better than controls $(P<0.005)$; and control early blastocysts were significantly better than morulae $(P<0.05)$.

(ii) Number of live fetuses: VS1 + VS11 with sucrose dilution was significantly better than VS1 + VS11 without sucrose dilution $(P<0.0001)$; controls were significantly better than VSI without sucrose dilution $(P<0.005)$; controls were significantly better than VSII without sucrose dilution $(P<0.01)$; and early blastocysts were significantly better than blastocysts $(P<0.005)$ and morulae $(P<0.01)$ when VSI and VS11 were diluted with sucrose.

VS was removed from the embryo with sucrose than when removed with medium (Table 3). The total implantations observed for embryos vitrified with VSII (which was diluted with or without sucrose) were significantly greater than those for untreated embryos and for those that were vitrified with VS1 (with or without dilution with sucrose). There were no differences between the total implantations observed for embryos vitrified with VS1 and those for untreated embryos. In the control group, the total implantation rate was significantly lower with morulae than with early blastocysts and blastocysts. In the test groups (embryos vitrified with VSI and VS1I), the total implantation rates were comparable for all developmental stages of day-4 embryos.

In summary embryo survival was better after vitrification with VS11 than with VS1 and, with either vitrification solution, embryo survival was improved by removal of the VS with sucrose.

The number of live fetuses formed following transfer of vitrified $\mathrm{SO}$ embryos was significantly greater for embryos from which the VS ( 1 and 11) were removed with sucrose than with medium. When VSI or VSII from SO embryos was removed with sucrose the number of live fetuses produced did not differ from that obtained from untreated SO embryos. Within the control group no differences among the three developmental stages of the day- 4 embryos were observed in the number of live fetuses. In groups vitrified with VS1 or VS11 which was removed with sucrose, early blastocysts formed significantly more live fetuses than blastocysts or morulae.

No differences were observed in the total number of implantations or number of live fetuses formed between $F_{1}$ control day-4 embryos and $F_{1}$ day-4 embryos vitrified with VS11 that was removed with sucrose (Table 4).

The total implantation rate and the number of live fetuses formed from SO mouse blastocysts vitrified with VS14 that was removed with sucrose were significantly lower than those of early blastocysts and morulae. There were no differences between vitrified early blastocysts and morulae (Table 5).

\section{Experiment 4: reproductive capability of mice derived from vitrified embryos}

Litters were produced by three out of five pairs of SO mice derived from day-4 embryos that were vitrified with VS1. The offspring comprised 17 males and 19 females. Litters were produced by four of five pairs of SO mice derived from day-4 embryos that were vitrified with VS11. The offspring comprised 
Table 4. Survival of day- $4 \mathrm{~F}_{1}{ }^{*}$ mouse embryos vitrified with VS11 when transferred to pseudopregnant C57BL/6 surrogates

\begin{tabular}{|c|c|c|c|c|c|}
\hline Treatment & $\begin{array}{c}\text { Stage of } \\
\text { development }\end{array}$ & $\begin{array}{l}\text { Number of } \\
\text { embryos } \\
\text { transferred }\end{array}$ & $\begin{array}{c}\text { Number of } \\
\text { live fetuses } \\
(\%)\end{array}$ & $\begin{array}{l}\text { Number of } \\
\text { resorptions } \\
(\%)\end{array}$ & $\begin{array}{l}\text { Total number of } \\
\text { implantations } \\
(\%)\end{array}$ \\
\hline \multirow[t]{4}{*}{ Untreated controls } & Blastocysts & 55 & $8(14.6)$ & $2(3.6)$ & $10(18.2)$ \\
\hline & Early blastocysts & 48 & $10(20.8)$ & $1(2.1)$ & $11(22.9)$ \\
\hline & Morulae & 32 & $5(15.6)$ & 0 & $5(15.6)$ \\
\hline & Total & 135 & $23(17.0)$ & $3(2.2)$ & $26(19.3)$ \\
\hline \multirow{4}{*}{$\begin{array}{l}\text { VS11 with } \\
\text { sucrose dilution }\end{array}$} & Blastocysts & 52 & $16(30.8)$ & $1(1.9)$ & $17(32.7)$ \\
\hline & Early blastocysts & 58 & $12(20.7)$ & $1(1.7)$ & $13(22.4)$ \\
\hline & Morulae & 33 & $11(33.3)$ & 0 & $11(33.3)$ \\
\hline & Total & 143 & $39(27.3)$ & $2(1.4)$ & $41(28.7)$ \\
\hline
\end{tabular}

${ }^{*} \mathrm{~F}_{1}$ mouse embryos were $((\mathrm{C} 57 \mathrm{BL} / 6 \times \mathrm{SJL}) \times$ Swiss Outbred $)$.

Summary of statistical analysis of number of total implantations and number of live fetuses: VS11 blastocysts were not significantly different from control blastocysts; VS11 early blastocysts were not significantly different from control early blastocysts; VS11 morulae were not significantly different from control morulae.

Table 5. Survival of Swiss Outbred mouse embryos vitrified with VS14 when transferred to pseudopregnant C57BL/6 surrogates

\begin{tabular}{llcccr}
\hline Treatment & \multicolumn{1}{c}{$\begin{array}{c}\text { Stage of } \\
\text { development }\end{array}$} & $\begin{array}{c}\text { Number of } \\
\text { embryos } \\
\text { transferred }\end{array}$ & $\begin{array}{c}\text { Number of } \\
\text { live fetuses } \\
(\%)\end{array}$ & $\begin{array}{c}\text { Number of } \\
\text { resorptions } \\
(\%)\end{array}$ & $\begin{array}{c}\text { Total number of } \\
\text { implantations } \\
(\%)\end{array}$ \\
\hline $\begin{array}{l}\text { VS14 with } \\
\text { sucrose dilution }\end{array}$ & Blastocysts & 37 & $2(5.4)$ & 0 & $2(5.4)$ \\
& Early blastocysts & 52 & $13(25.0)$ & $2(3.9)$ & $15(28.9)$ \\
& Morulae & 30 & $10(33.3)$ & $4(13.3)$ & $14(46.7)$ \\
& Total & 119 & $25(21.0)$ & $6(5.0)$ & $31(26.1)$ \\
\hline
\end{tabular}

Summary of statistical analysis: (i) Total number of implantations: blastocysts versus early blastocysts, $P<0.05$; blastocysts versus morulae, $P<0.0005$; and early blastocysts versus morulae, not significant.

(ii) Number of live fetuses: blastocysts versus early blastocysts, $P<0.05$; blastocysts versus morulae, $P<0.01$; and early blastocysts versus morulae, not significant.

21 males and 10 females. All the offspring appeared to be normal.

\section{Discussion}

The survival rates of vitrified embryos in vitro were excellent and showed no clear differences among VS when they were used with day-4 morulae, early blastocysts and blastocysts. However, earlier developmental stages were less tolerant of the VS and differences among the VS became apparent in the survival rates. With a few exceptions failure to achieve good survival after vitrification was related to toxicity of the VS. The viability of eight-cell embryos was reduced by exposure to VS11 but not by vitrification in VS11. Although this is difficult to explain, it is worth noting that there was little difference $(P=0.04)$ between the survival rates of embryos exposed to and those vitrified in VS11. Furthermore the high survival rate of eight-cell embryos vitrified in VSI1 in Expt 2 (90.9\%) suggests that reduced survival observed in Expt $I$ is an aberration due perhaps to experimental error. Clearly VS14 was the least toxic of the vitrification solutions tested followed by VS11 and then VSI.

There are two components of cryoprotectant toxicity: chemical toxicity and toxicity due to osmotic stress. Ali (1992) found that of the permeating cryoprotectants present in the VS that were tested here, glycerol is the more toxic. It is present at 2.5, 1.8 and $0 \mathrm{~mol} \mathrm{I}^{-1}$ in VS1, VSII and VSI4, respectively, that is, in the same descending order as the toxicity of the VS. Although toxicity studies suggested that these molarities of glycerol are not toxic to embryos exposed for a brief time, it is possible that toxicity is enhanced under conditions of vitrification. Furthermore, the marginal increase in toxicity of the VS resulting from increasing concentrations of glycerol may be critical for the embryos at less tolerant developmental stages. An additional factor contributing to the decreased survival of early preimplantation stages of embryos with increasing glycerol content of the VS may be the lower permeability coefficient of these stages to glycerol (Mazur et al, 1976). An inexplicable exception was the failure of $F_{1}$ one-cell embryos to survive vitrification in VS11, whereas $67 \%$ survived vitrification in VS1. 
Osmotic stress imposed by the VS is a function of the osmolality of the VS. The calculated osmolalities of the three VS are 12.17, 12.74 and 10.39 osmoles for VSI, VSII and VS14, respectively. Thus it may be concluded that difference in osmotic stress is not the reason for the lower toxicity of VSII compared with VS1. Although the osmolality of VS14 is lower than that of VS1 and VS11, the significance of this in relation to the lower toxicity of VSI4 is not clear. However, it is interesting, and probably important, in considering further experiments, that the addition of $1 \mathrm{~mol}$ sucrose $\mathrm{I}^{-1}$ allows the concentration of ethylene glycol to be reduced to $5.5 \mathrm{~mol} \mathrm{l}^{-1}$ without loss of vitrifying capacity. Neither $5.5 \mathrm{~mol}$ ethylene $\mathrm{l}^{-1}$ nor $1 \mathrm{~mol}$ sucrose $1^{-1}$ will vitrify on its own.

The data on survival of vitrified embryos when transferred to pseudopregnant recipients confirmed the efficacy of the three VS under test. Although VS14 was not included with VS1 and VS11 in viability experiments in vivo, the data of in vivo viability of day-4 embryos vitrified with VS14 strongly suggest that VSI4 may result in better survival in vivo than would the other VS. The results of transfer experiments in terms of both total implantations and live fetuses were not remarkable, but comparisons within the experiments remain valid. VSII was superior to VSI in the total number of implantations, but there was no difference in the number of live fetuses from embryo transfer. These results were produced with one-step exposure of the embryos to the vitrification medium at room temperature $\left(25^{\circ} \mathrm{C}\right)$. It is interesting that Kasai et al. (1990), after testing cryoprotectants for both vitrification and toxicity, also found that ethylene glycol was the permeating cryoprotectant of choice. Most previous investigators attached major importance to vitrification in the selection of permeating cryoprotectant and resorted to stepwise exposure of the embryos to the vitrification medium at low temperature (commonly $4^{\circ} \mathrm{C}$ ) to circumvent embryotoxicity.

Sucrose dilution of the VS was beneficial to both the total number of implantations and the number of live fetuses developed. The beneficial effect of sucrose can be attributed to the prevention of excessive swelling of the embryos which may occur when dilution is by exposure to physiological medium (Leibo and Mazur, 1978; Kasai et al., 1980). When VS was removed with sucrose, the vitrified embryos produced more total implantations than did untreated embryos and the same number of live fetuses. The fewer total implantations from untreated embryos may have been due to the experimental procedure, whereby the control embryos were held under laboratory conditions, whereas embryos from the same population were exposed to VS and vitrified then warmed and put through the VS dilution procedure before transfer. When $F_{1}$ embryos were used, there was again no difference in the number of live fetuses produced from vitrified (VSI1) and untreated embryos. In a separate experiment, VS14 produced very similar results. Clearly the vitrification solutions formulated and applied in experiments described here are excellent for cryopreservation of mouse embryos.

There was a lack of consistency between the in vitro and in vivo experiments on the effect of using sucrose in the removal of VS from vitrified embryos after warming. There was no significant effect of removal by sucrose on survival of day-4 embryos (blastocysts, early blastocysts and morulae) in vitro, but there was a significant benefit in using sucrose when embryos were transferred to pseudopregnant recipients. The reasons for this difference are not apparent but they no doubt reflect the different end-points in the two methods of assessment. In vitro assessment was based on development of blastocysts that appear normal but with no assurance that they can develop to normal fetuses. Disparity between the two end-points may result from different survival rates of subpopulations of cells (e.g. trophoblast or inner cell mass). The apparent better survival of two-cell embryos when diluted directly with medium than when diluted with sucrose may reflect the small number of two-cell embryos used in this experiment; alternatively it may indicate that $1 \mathrm{~mol}$ sucrose $1^{-1}$ is toxic to two-cell mouse embryos.

The rate of viability observed in vivo in the present study is lower than in other reports (Kasai et al., 1990; Ishimori et al., 1992) on viability of vitrified day-4 embryos. Kasai et al. (1990) reported a $51 \%$ live birth and Ishimori et al. (1992) reported development of $34-36 \%$ of vitrified embryos to live fetuses. The low rate of development in vivo of both vitrified and control embryos in the experiments described here could be due to the inhalation anaesthetic (Penthrane) used, or to the embryo transfer technique. The latter was performed with a drawn-out fine glass pipette attached by plastic tubing to a syringe drive, which was activated to expel the embryos into the uterus. The conventional technique of embryo transfer with mouth-piece and rubber tubing could not be performed owing to the sensitivity of the investigator to mouse fur and urinary products, and the need to wear a mask.

In the experiments described here, there was no attempt to equilibrate completely the embryos with cryoprotectant and yet high rates of embryo survival resulted after vitrification. The degree of permeation of embryonic cells by the VS is unknown, but the studies on changes in embryo volume following exposure to VS (Ali, 1992) indicate that, after the short duration of exposure used here, there could have been very little permeation. The immediate response of the embryos to exposure to VS is dehydration because of the high concentration of the cryoprotectants and their low permeativity relative to water. Consequently, the concentration of solutes and macromolecules within the blastomeres is increased markedly and probably contributes to intracellular vitrification when the embryos are subjected to rapid cooling by plunging them into liquid nitrogen. Extracellular vitrification is promoted by the high concentration of cryoprotectants in the VS which may also have a role in protecting cell membranes. This discussion leads to speculation on what may occur when embryos are cryopreserved by rapid cooling in medium that is not sufficiently concentrated to vitrify (Shaw and Trounson, 1989; Wilson and Quinn, 1989; Li and Trounson, 1991; Shaw et al., 1991). It is arguable that in these procedures intracellular vitrification occurs owing to concentration of solutes resulting from dehydration of the blastomeres. Rall and Polge (1984) demonstrated intracellular vitrification in embryos dehydrated by slow cooling in 1.5 mol glycerol $\mathrm{l}^{-1}$. However, mouse embryos cannot be frozen rapidly or vitrified with 1 mol sucrose $1^{-1}$ (J. Ali and J. N. Shelton, unpublished observations) or 2 mol sucrose $1^{-1}$ (Ali, 1992) without complete loss of viability, suggesting that permeating cryoprotectants are essential for survival of frozen and vitrified embryos. 
Mice derived from day-4 SO mouse embryos vitrified with VSI and VS1I could reproduce and teratogenic effects were not seen in any of the offspring. Vitrification can therefore be routinely used to cryopreserve mouse embryos.

We thank W. K. Whitten for his assistance in embryo transfer procedures.

\section{References}

Ali J (1992) Factors Affecting Ultrarapid Vitrification and Cryopreservation of Embryos PhD Thesis, Australian National University, Canberra

Hammond J, Jr (1949) Recovery and culture of tubal mouse ova Nature 163 $28-29$

Ishimori H, Takahashi $\mathbf{Y}$ and Kanagawa $\mathbf{H}$ (1992) Viability of vitrified mouse embryos using various cryoprotectant mixtures Theriogenology 37 481-487

Kasai M, Niwa K and Iritani A (1980) Survival of mouse embryos frozen and thawed rapidly Joumal of Reproduction and Fertility 59 51-56

Kasai M, Komi JH, Takakamo A, Tsudera H, Sakurai T and Machida T (1990) A simple method for mouse embryo cryopreservation in a low toxicity vitrification solution, without appreciable loss of viability Journal of Reproduction and Fertility 89 91-97

Leibo SP and Mazur P (1978) Methods for the preservation of mammalian embryos by freezing. In Methods in Mammalian Reproduction pp 179-201 Ed. JC Daniel, Jr. Academic Press, New York

Li R and Trounson AO (1991) Rapid freezing of the mouse blastocyst: effects of cryopreservation and of time and temperature of exposure to cryoprotectant before direct plunging into liquid nitrogen Reproduction Fertility and Development 3 175-183
Luyet B (1937) The vitrification of organic colloids and of protoplasm Biodynamica 1 I-14

McLaren A and Michie D (1956) Studies on the transfer of fertilized mouse eggs to uterine foster-mothers. I. Factors affecting the implantation and survival of native and transferred eggs Journal of Experimental Biology 33 394-416

Mazur P, Rigopoulos N, Jackowski S and Leibo SP (1976) Preliminary estimates of the permeability of mouse ova and early embryos to glycerol Biophysical Journal 16232 (Abstract)

Rall WF (1987) Factors affecting the survival of mouse embryos cryopreserved by vitrification Cryobiology 24 387-402

Rall WF and Fahy GM (1985) Vitrification: a new approach to embryo cryopreservation Theriogenology 23220 (Abstract)

Rall WF and Polge C (1984) Effect of warming rate on mouse embryos frozen and thawed in glycerol Journal of Reproduction and Fertility 70 285-292

Rall WF, Meyer TK and Leibo SP (1986) Effect of warming conditions on the survival of mouse embryos cryopreserved and diluted by a one-step straw procedure Theriogenology 25186 (Abstract)

Scheffen B, Van der Zwalmen P and Massip A (1986) A simple and efficient procedure for preservation of mouse embryos by vitrification Cryo-letters 7 $260-269$

Shaw JM and Trounson AO (1989) Effect of dimethyl sulfoxide and protein concentration on the viability of two-cell mouse embryos frozen with a rapid freezing technique Cryobiology 26 413-421

Shaw JM, Diotellevi L and Trounson AO (1991) A simple rapid 4.5M dimethyl sulphoxide freezing technique for the cryopreservation of one-cell to blastocyst stage preimplantation mouse embryos Reproduction Fertility and Development 3 621-626

Whitten WK (1957) Effect of exteroreceptive factors on the oestrous cycle of mice Nature 1801436

Whitten, WK (1971) Nutrient requirements for the culture of preimplantation embryos in vitro Advances in Bioscience 6 129-141

Wilson L and Quinn P (1989) Development of mouse embryos cryopreserved by an ultrarapid method of freezing Human Reproduction 4 86-90 